Nov. 14th, 1894, I operated and removed the cyst entire, it being of about the size of one's two fists. She made an uninterrupted recovery and left the home on Dec. 14th; a month after the operation.

The interest in the case lies in the fact of the cyst rupturing, without the slightest symptom, the quick recovery of the patient, the gradual reappearance of the cyst, its gradual enlargement, and on its removal the plainly marked scar on its anterior surface running diagonally across from left to right. No doubt the patient being anæsthetised when the cyst ruptured prevented shock, but on her recovering from the anæsthetic there was not the slightest sign of anything wrong, no peritoneal irritation, \&c., and she nursed the child for six months. She is still alive and well.

I am, Sir, yours faithfully,

Queen's gate, S.W., Feb. 14th, 1910. SIDNEY HARVEY.

\section{THE POOR-LAW MEDICAL SERVICE.}

\section{To the Editor of THE LANCET.}

SIR,-I should like through the medium of THE LANCET to draw attention to a few crying grievances of the Poor-law infirmary medical officers in London, with at the same time suggestions for their redress. Under present conditions the assistant medical officer is a servant of the infirmary committee of the local guardians, who may or may not get on well with a professional man, but who have naturally, in most instances, very little idea of the actual scope of his work. There is no security of tenure of office, there is understaffing such as would not be tolerated in any other medical service, and the pay is inadequate. The work is exacting if it is to be done properly, the infirmaries being like large general hospitals without their special departments.

There is one safeguard provided, and that is appeal to the Local Government Board, which is an extremely busy department with no time to listen to complaints such as this coming from an individual. The guardians are at present having things their own way and behaving in the most approved demagogic manner. They are kicking efficient men out of the service; they are browbeating others; they are taking to appointing their assistant medical officers for a limited number of years (three is a favourite number). The result will be that when superintendentships in the service become vacant they will fall to the highly trained officers of the fever hospital service and not to the half-trained Poor-law medical officers. This Juggernaut career of the guardians will continue just so long as the medical officers will put up with it.

As we gaze with envy at our more fortunate brethren in the service of the Metropolitan Asylums Board the thought arises that the remedy lies in the organisation of such another board for the control of the Poor-law infirmaries of London. Our most immediate desire is to be delivered out of the hands of the guardians, and this can be done only by a concerted effort on our part. When this was accomplished there could be established a proper infirmary medical service, with security of tenure, promotion, adequate pay and staffing, and a homogeneity that would make for improved work clinically and in research instead of the present guardian-ridden heterogeneous mass of atoms that does not even produce statistics. In other words, a service could be formed to which a man could devote his life, working unhampered by petty spite and wire-pulling.

I am, Sir, yours faithfully, ALAN RANDLE, M.D. Lond.

St. Marylebone Infirmary, Notting Hill, W., Feb. 21st, 1910.

\section{THE AXIAL NOTATION OF CYLINDRICAL LENSES.}

To the Editor of THE LANCET.

StR,-Mr. Percy Dunn, in the description of his ingenious application of the compass points to the prescription of cylindrical lenses, finds it difficult to understand why the horizontal base-line has been selected as the meridian of departure in the enumeration of angular values. I believe that it was selected by the Optical Standards Committee of the Optical Society as being in accordance with the practice obtaining in every department of applied geometry ur physical measurement involving the use of the protractor.
The old scale is that of " nasal zeros." There is a horizontal base-line for each eye, but the degree enumeration proceeds for the right eye down and to the right, while in the left eye it is down and to the left. Hence the need of qualifying expressions, such as "down and out," \&c.

Mr. Dunn has improved on that because his chart is applicable to either eje, but it is not so good as the Optical Society's since it has a duplicate set of numerals, necessitating qualification. He has, for example, two angles of $30^{\circ}$, and the required one has to be specified N.E. or N.W. The Optical Society's notation reads down and to the left for both ezes. In other words, one has only to apply the present left eye scale of the old style to both eyes to have the new. In this, a meridian of $30^{\circ}$ is that and that alone. It needs no specification of eye, direction, or compass bearing. In case-recording that is important, and my view that it is the simplest and best is based on some years of practical use as well as on theoretical considerations.

I am, Sir, yours frithfully,

William ETTLES, M.D. Aberd., F.R.C.S. Edin.

Wimpole-street, W., Feb. 19th, 1910.

\section{THE EARLY DIAGNOSIS OF CANCER OF THE STOMACH.}

\section{To the Editur of THE LANCET.}

SIR,- I am much obliged to Dr. W. Gordon for his complimentary allusions to myself, and for the kind manner in which he tries to explain away the significance of the facts I brought before the Hunterian Society in a lecture which was published in your issue of Feb. 12th. They were very simple. I dealt with only one special class of patients, those suffering from cancer of the stomach. That 60 per cent. of these gave a history such as is ordinarily accepted as diagnostic of pre-existing ulcer of the stomach is a fact. Other observers have found the same, and our conclusions are being confirmed every day by the results of the study of the pathology of the living. Whether a collective investigation of all sorts of gastric cases, unselected, with or without indications for operation, by any number of ob. servers, as recommended by Dr. Gordon, would give a valuable result is a matter for opinion, but it can have no bearing upon this.

Dr. Gordon's illustration of the ophthalmologist is singularly inapposite. There might have been some point in it if I had written a treatise on gastric ulcer, and asserted that cancer was an almost invariable accompaniment of it but then I did not do this. What I maintain is that cancer in the stomach originates almost always on the site of some unhealed ulcer or irritated scar, as it notoriously does in all other parts of the body; and that now that this site can be seen with a gastroscope there is but one thing to do, and that is remove the point of origin in time. The doctrine is perfectly logical and is in accordance with the facts; but I suppose it will not meet with general acceptance until the lessons of post-mortem pathology are given their true value, and those learnt from timely operation upon the living are appreciated as they deserve to be.

I am, Sir, yours faithfully,

Wimpole-street, W., Feb. 19th, 1910 . C. Mansell Moullin.

\section{To the Editor of THE LANCET.}

Sir,-Will you allow me to comment on two matters arising out of my paper, read before the Surgical Section of the Royal Society of Medicine and reported in your current issue? In criticising my paper Mr. H. J. Paterson misunderstood my statement about the cause of death I showed that of 127 cases in which the cause of death after gastrectomy had been reported 97 were due to some form of peritonitis, chiefly from leakage. These figures are collected from a number of different surgeons who practise "the improved technique of the present day." It is not a question of what any self-respecting surgeon would dream of doing, but merely an actual narration of what modern surgeons have done.

Dr. W. Gordon writes strongly differing from the views expressed by Mr. Moullin and Mr. Moynihan, who think that a large proportion of cases of cancer of the stomach are preceded by ulcer. A recent very careful paper in the December 